\title{
EDUCAÇÃO NO CENTRO DE MATERIAIS E ESTERILIZAÇÃO: REVISÃO INTEGRATIVA
}

\author{
Education at the Materials and Sterilization Center: an Integrative Review
}

\author{
Educación en el centro de materiales y esterilización: revisión integrativa \\ Carolina Gerhardt Primaz ${ }^{1 *} \oplus$, Rúbia Knobeloch dos Santos ${ }^{\circledR} \odot$, Jessyca Zanetti Malgor Oliveira ${ }^{3} \oplus$, \\ Paôla Maros Heinen ${ }^{3} \odot$, Rita Catalina Aquino Caregnato ${ }^{4}$ ๑
}

RESUMO: Objetivo: Analisar as publicações dos últimos cinco anos com temas sobre educação em Centro de Materiais e Esterilização. Método: Revisão integrativa com busca nas bases de dados: Literatura Latino-Americana e do Caribe em Ciências da Saúde, Medical Literature Analysis and Retrieval System Online, Scopus Info Site, Web of Science, Cumulative Index to Nursing and Allied Health Literature via EBSCOhost, Cochrane e Biblioteca Virtual em Saúde. Resultados: Selecionados dez artigos, todos consideraram indispensáveis a atualização constante e a disponibilização de recursos mínimos para realização das atividades profissionais com melhorias na prática clínica. Quatro publicações identificaram as percepções quanto à educação, lacunas de conhecimento e oportunidades de desenvolvimento. Estratégias educacionais utilizadas: educação permanente com materiais didáticos, aplicação de treinamento teórico e prático, reestruturação dos processos com treinamento técnico, programas de mentoria educativa in loco e autorreflexão profissional sobre melhores práticas aplicadas nos processos de trabalho. Conclusão: As publicações reforçam a importância de intervenções educativas para capacitação profissional, domínio de novas tecnologias, prestação de serviço de qualidade e impacto favorável na eficácia do processamento de produtos para a saúde. Palavras-chave: Esterilização. Educação. Segurança do paciente.

ABSTRACT: Objective: To analyze the publications of the last five years about education at the Materials and Sterilization Center. Method: Integrative review with search in databases: Latin American and Caribbean Health Sciences Literature, Online Medical Literature Analysis and Retrieval System, Scopus Info Site, Web of Science, Cumulative Index to Nursing and Allied Health Literature via EBSCOhost, Cochrane, and Virtual Health Library. Results: Ten articles were selected, all of them considering it essential to constantly update and make available minimum resources to carry out professional activities with improvements in clinical practice. Four publications identified perceptions of education, knowledge gaps and development opportunities. The educational strategies used were: continuing education with teaching materials, application of theoretical and practical training, restructuring of processes with technical training, on-site educational mentoring programs and professional self-reflection on best practices. Conclusion: The publications reinforce the importance of educational interventions for professional training, with view to them mastering new technologies, providing quality services and having a favorable impact on the effectiveness of health products' processing.

Keywords: Sterilization. Education. Patient safety.

RESUMEN: Objetivo: Analizar las publicaciones de los últimos cinco años con temas de educación en el Centro de Materiales y Esterilización. Método: Revisión integradora con búsqueda en bases de datos: Literatura de Ciencias de la Salud de América Latina y el Caribe, Medical Literature Analysis and Retrieval System Online, Scopus Info Site, Web of Science, Cumulative Index to Nursing and Allied Health Literature via EBSCOhost, COCHRANE y Biblioteca Virtual en Salud. Resultados: Se seleccionaron 10 artículos, todos considerados imprescindibles a la constante actualización y disponibilidad de recursos mínimos para realizar actividades profesionales con mejoras en la práctica clínica. Cuatro publicaciones identificaron percepciones de educación, brechas

'Mestranda do Programa de Pós-Graduação em Ensino na Saúde, Universidade Federal de Ciências da Saúde de Porto Alegre (UFCSPA) - Porto Alegre (RS), Brasil. ${ }^{2}$ Enfermeira pela UFCSPA. Enfermeira residente do Programa de Terapia Intensiva da UFCSPA / Santa Casa de Misericórdia de Porto Alegre - Porto Alegre (RS), Brasil. ${ }^{2}$ Graduanda em Enfermagem, UFCSPA - Porto Alegre (RS), Brasil.

«Doutora em Educação pela Universidade Federal do Rio Grande do Sul. Professora adjunta do Departamento de Enfermagem da UFCSPA - Porto Alegre (RS), Brasil.

*Autora correspondente: carolinagp@ufcspa.edu.br

Recebido: 06/11/2020 - Aprovado: 22/07/2021

https://doi.org/10.5327/Z1414-4425202100030007 
de conocimiento y oportunidades de desarrollo. Estrategias educativas utilizadas: formación continua con material didáctico, aplicación de formación teórica y práctica, reestructuración de procesos con formación técnica, programas de tutoría educativa presencial y autorreflexión profesional sobre las mejores prácticas aplicadas en los procesos de trabajo. Conclusión: Las publicaciones refuerzan la importancia de las intervenciones educativas para la formación profesional, el dominio de las nuevas tecnologías, la prestación de servicios de calidad y el impacto favorable en la efectividad del procesamiento de los productos sanitarios.

Palabras clave: Esterilización. Educación. Seguridad del paciente.

\section{INTRODUÇÃO}

O Centro de Materiais e Esterilização (CME) é uma área com grande especificidade e importância dentro dos serviços de saúde (SS), fundamental para sua estrutura e funcionamento. Nesse setor, são realizadas práticas diversificadas que requerem conhecimento especializado, com a finalidade de fornecer produtos para a saúde (PPS) processados de forma adequada, garantindo a segurança na prestação do cuidado assistencial ao paciente ${ }^{1,2}$.

As atividades desenvolvidas no CME compreendem recebimento de materiais sujos/contaminados, limpeza, inspeção, preparo, desinfecção e esterilização, armazenamento e distribuição ${ }^{3}$. Todos os processos realizados nesse setor têm a finalidade de prevenir as infecções relacionadas à assistência à saúde (Iras), seguindo as evidências científicas para uma prática de qualidade no preparo dos $\mathrm{PPS}^{4}$.

Os profissionais que trabalham no CME não desempenham apenas atividades rotineiras e repetitivas, mas fornecem apoio técnico indispensável ao garantir materiais livres de contaminação e seguros para assistência de pacientes ${ }^{5,6}$. Nesse sentido, os profissionais devem compreender a relevância da condução dos processos envolvidos e a importância da sua atuação. É imprescindível acompanhar o avanço tecnológico e as transformações ocorridas nos outros setores da saúde, como o desenvolvimento de novos instrumentais e técnicas cirúrgicas ${ }^{2,7,8}$.

A partir da compreensão dos processos de trabalho e do reconhecimento das atividades, cada vez mais complexas e sofisticadas, exercidas no CME, eleva-se a importância de recursos humanos qualificados e motivados ${ }^{1}$. Por isso, estratégias de educação que reforcem o aprimoramento dos profissionais inseridos no contexto do CME são importantes para garantir a qualidade e a segurança do serviço prestado nesse setor, considerando a carência de especificidade durante a formação profissional ${ }^{9,10}$.

Portanto, a implementação de atividades de educação continuada (EC) e educação permanente em saúde (EPS) é fundamental para promover mudanças nos processos de trabalho e suas relações entre os sujeitos e o ambiente, ampliando-se os espaços de aprendizagem e contribuindo para o desenvolvimento dos profissionais e do próprio serviço ${ }^{4}$.

Diante do cenário traçado, emergiu o interesse em pesquisar o tema educação no CME. Pretende-se fornecer um panorama atualizado em relação à produção de conhecimento na área de educação direcionada ao profissional que trabalha na linha de frente no processamento de PPS.

\section{OBJETIVO}

Analisar as publicações dos últimos cinco anos com temas sobre educação em CME.

\section{MÉTODO}

Trata-se de uma revisão integrativa, que possibilita sintetizar e analisar o conhecimento científico disponível para busca de dados que respondam a uma questão de pesquisa ${ }^{11,12}$.

Para atender ao rigor metodológico, o artigo seguiu as seis etapas:

- identificação do tema e seleção da questão de pesquisa;

- estabelecimento dos critérios de inclusão e exclusão;

- identificação dos estudos pré-selecionados e selecionados;

- categorização dos estudos selecionados;

- análise e interpretação dos resultados;

- apresentação da revisão ou síntese do conhecimento ${ }^{11,12}$.

Para o presente estudo, formulou-se a seguinte questão de pesquisa: o que existe publicado sobre educação dos profissionais que atuam no CME?

Para delimitação da busca, foram definidos como critérios de inclusão artigos científicos publicados na íntegra, de 
janeiro de 2015 a outubro de 2020, com livre acesso online, que abordassem a questão de pesquisa, independentemente de sua tipologia. Os critérios de exclusão foram: publicações classificadas como editorial, cartas, dissertações, teses, manuais e protocolos.

Os dados foram coletados nas bases de dados científicas online: Literatura Latino-Americana e do Caribe em Ciências da Saúde (Lilacs), Medical Literature Analysis and Retrieval System Online (Medline / PubMed), Scopus Info Site (Scopus), Web of Science, Cumulative Index to Nursing and Allied Health Literature (CINaHL) via EBSCOhost, Cochrane e na Biblioteca Virtual em Saúde (BVS).

A busca dos artigos nas bases ocorreu no período de 20 de agosto até 7 de outubro de 2020. Foram definidos os descritores controlados obtidos nos Descritores em Ciências da Saúde (DeCS) e no Medical Subject Headings (MeSH): sterilization, education, continuing, professional e medical devices. Também foram utilizados descritores não controlados: central sterile supply department, sterile processing department e sterilizing practices. Os descritores definidos para a busca foram combinados entre si pelos operadores booleanos "AND" e/ ou "OR" e testados em diferentes associações, resultando nas sequências de combinações apresentadas no Quadro 1.

Para restrição dos resultados que atendessem à pergunta de pesquisa, elegeu-se a quarta combinação e, após a aplicação dos filtros, foram identificados 13.346 artigos. Mediante triagem pela leitura dos títulos, restaram 264 artigos. Desses, foram eliminados 213 após a leitura dos resumos por apresentar um ou mais critérios de exclusão e 30

Quadro 1. Descritores combinados com operadores booleanos utilizados nas etapas de busca dos artigos.

\begin{tabular}{|l|c|}
\hline $\begin{array}{l}\text { Etapas } \\
\text { da busca }\end{array}$ & $\begin{array}{c}\text { Combinações dos descritores } \\
\text { com operadores booleanos }\end{array}$ \\
\hline $1^{\mathrm{a}}$ & $\begin{array}{c}\text { Sterilization AND Education OR Continuing } \\
\text { Education }\end{array}$ \\
\hline $2^{\mathrm{a}}$ & $\begin{array}{c}\text { Sterilization OR Medical devices OR Central sterile } \\
\text { supply department AND Education OR Continuing } \\
\text { Education OR Professional Education }\end{array}$ \\
\hline $3^{\mathrm{a}}$ & $\begin{array}{c}\text { Sterilization OR Medical devices OR Central } \\
\text { sterile supply department OR Sterile processing } \\
\text { department AND Education OR Continuing } \\
\text { Education OR Professional Education }\end{array}$ \\
\hline $4^{\mathrm{a}}$ & $\begin{array}{c}\text { Sterilization OR Medical devices OR Central } \\
\text { sterile supply department OR Sterile processing } \\
\text { department OR Sterilizing practices AND Education } \\
\text { OR Continuing Education OR Professional Education }\end{array}$ \\
\hline
\end{tabular}

por serem repetidos em diferentes bases de dados. Depois da leitura dos artigos na íntegra, foram excluídos mais 11 por não atender ao tema de pesquisa, e mantidos os dez artigos que se enquadraram nos critérios definidos previamente e responderam à pergunta norteadora, constituindo, portanto, a amostra final.

O estudo cumpriu as etapas recomendadas a serem seguidas pelo instrumento Preferred Reporting Items for Systematic Reviews and Meta-Analyses (Prisma) ${ }^{13}$, conforme a Figura 1.

Para a coleta de dados, utilizou-se um instrumento detalhado contendo as variáveis: título, periódico, autores, ano de publicação, objetivos, métodos e resultados. Na etapa seguinte, realizou-se a análise e a síntese dos artigos obtidos de forma descritiva.

Os artigos foram avaliados e classificados quanto ao seu rigor científico, conforme as características de cada estudo, possibilitando uma classificação por nível de evidência. Nessa etapa, foi utilizado um instrumento baseado na Rating System for the Hierarchy of Evidence for Intervention/Treatment Question ${ }^{14}$ para classificação do nível de evidência (NE) dos estudos, apresentada no Quadro 2.

\section{RESULTADOS}

Em relação às dez publicações selecionadas, $50 \%$ foram publicadas em periódicos estrangeiros. Desses, cinco são provenientes do Brasil, dois da África, um da Austrália, um dos Estados Unidos e um da China. Os periódicos nos quais os artigos foram publicados são: dois na Revista SOBECC e dois na Revista de Enfermagem da Universidade Federal de Pernambuco (UFPE) On Line, e os demais publicados um em cada periódico a seguir: Revista Mineira de Enfermagem (Reme); Journals Elsevier - Infection, Disease \& Health; Surgical Infections; BMC Health Services Research; Antimicrobial Resistance and Infection Control; PlosOne.

Quanto ao delineamento da pesquisa, obtiveram-se: seis estudos exploratórios descritivos, uma revisão de literatura, dois relatos de experiência e um estudo de coorte. Em conformidade com Rating System for the Hierarchy of Evidence for Intervention/Treatment Question ${ }^{14}$, classificou-se: um artigo com nível de evidência 4 , um com nível de evidência 5 , seis com nível de evidência 6 e dois com nível de evidência 7 . O Quadro 3 apresenta o perfil dos artigos.

O Quadro 4 apresenta a síntese com os respectivos objetivos e resultados dos estudos incluídos na presente revisão integrativa. 


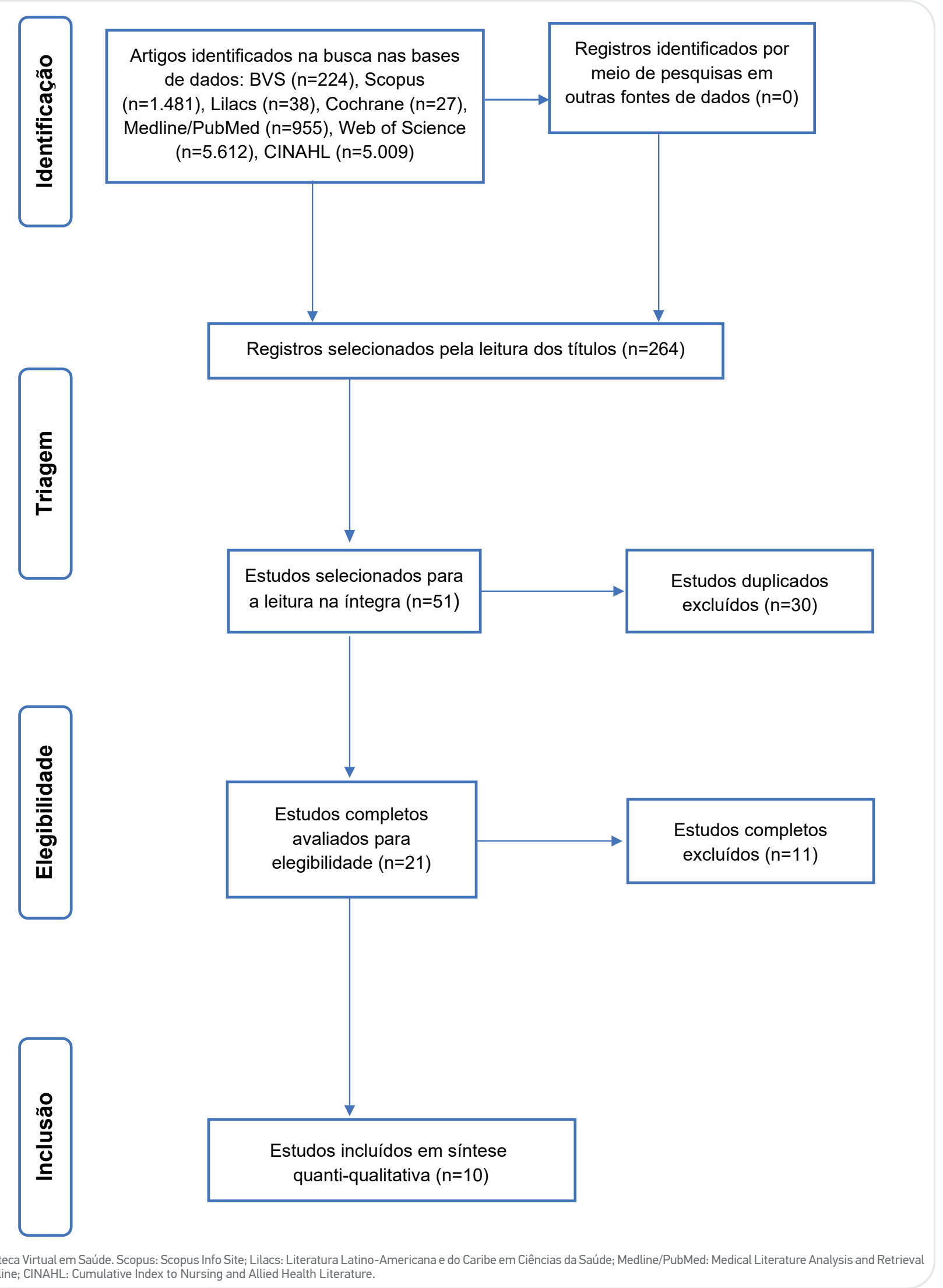

BVS: Biblioteca Virtual em Saúde. Scopus: Scopus Info Site; Lilacs: Literatura Latino-Americana e do Caribe em Ciências da Saúde; Medline/PubMed: Medical Literature Analysis and Retrieval System Online; CINAHL: Cumulative Index to Nursing and Allied Health Literature.

Figura 1. Fluxograma de seleção dos estudos sobre metodologias utilizadas para educação em Centro de Material e Esterilização. 
Quadro 2. Classificação do nível de evidência.

\begin{tabular}{|c|c|}
\hline \multicolumn{2}{|c|}{$\begin{array}{l}\text { Escala Rating System for the Hierarchy of Evidence for } \\
\text { Intervention/Treatment Questions }\end{array}$} \\
\hline NE1 & $\begin{array}{c}\text { Evidências de revisões sistemáticas ou meta-análise } \\
\text { de ensaios clínicos randomizados }\end{array}$ \\
\hline NE2 & Evidências de ensaios clínicos randomizados \\
\hline NE3 & Evidências de ensaios clínicos sem randomização \\
\hline NE4 & Evidências de caso-controle e estudos de coorte \\
\hline NE5 & $\begin{array}{l}\text { Evidências de revisões sistemáticas de estudos } \\
\text { descritivos e qualitativos }\end{array}$ \\
\hline NE6 & Evidências de estudos descritivos ou qualitativos \\
\hline NE7 & $\begin{array}{l}\text { Evidências de opinião de autoridades/relatórios de } \\
\text { experts }\end{array}$ \\
\hline
\end{tabular}

Fonte: Escala Rating System for the Hierarchy of Evidence for Intervention/Treatment Question'14.

\section{DISCUSSÃO}

O uso de estratégias educativas para qualificação dos profissionais que atuam no processamento de PPS tem sido de interesse nacional e internacional. As intervenções se intensificaram na última década, em consonância com a Organização Mundial da Saúde (OMS), que recomenda insistentemente a implementação de programas de educação continuada como iniciativa essencial para desenvolver as competências profissionais e elevar as práticas dos procedimentos assistenciais ${ }^{2}$, garantindo mais confiabilidade e segurança na prevenção de Iras.

As ações de ensino e aprendizagem empregadas para formação e capacitação dos profissionais, além de

Quadro 3. Caracterização do perfil dos artigos sobre educação em Centro de Material e Esterilização publicados em periódicos entre 2015 e 2020.

\begin{tabular}{|c|c|c|c|c|}
\hline Autores, ano e país & Título & Periódico & Delineamento & Nível de evidência \\
\hline $\begin{array}{l}\text { Athanázio e Cordeiro, } \\
2015^{15} \\
\text { Brasil }\end{array}$ & $\begin{array}{l}\text { Educação permanente a } \\
\text { trabalhadores da central de } \\
\text { material e esterilização }\end{array}$ & $\begin{array}{l}\text { Revista de } \\
\text { Enfermagem UFPE On } \\
\text { Line }\end{array}$ & $\begin{array}{l}\text { Qualitativo } \\
\text { exploratório descritivo }\end{array}$ & NE6 \\
\hline $\begin{array}{l}\text { Tolson e Friedewald, } \\
2016^{16} \\
\text { Austrália }\end{array}$ & $\begin{array}{l}\text { Beyond the patient zone: } \\
\text { improving hand hygiene } \\
\text { performance in a Sterilising } \\
\text { Services Department }\end{array}$ & $\begin{array}{c}\text { Journals Elsevier - } \\
\text { Infection, Disease \& } \\
\text { Health }\end{array}$ & Relato de experiência & NE7 \\
\hline $\begin{array}{l}\text { Farias et al., } \\
2016^{17} \\
\text { Brasil }\end{array}$ & $\begin{array}{c}\text { Continuing education in a } \\
\text { material and sterilization } \\
\text { center: perception of the } \\
\text { nursing team }\end{array}$ & $\begin{array}{l}\text { Revista de } \\
\text { Enfermagem UFPE On } \\
\text { Line }\end{array}$ & $\begin{array}{l}\text { Tranversal descritivo } \\
\text { quantitativo qualitativo }\end{array}$ & NE6 \\
\hline $\begin{array}{l}\text { Paula et al., } \\
2017^{18} \\
\text { Brasil }\end{array}$ & $\begin{array}{l}\text { Elaboration of didactic } \\
\text { material for processing } \\
\text { health products in primary } \\
\text { health care units }\end{array}$ & Revista SOBECC & Relato de experiência & NE7 \\
\hline $\begin{array}{l}\text { Bugs et al., } \\
2017^{19} \\
\text { Brasil }\end{array}$ & $\begin{array}{l}\text { Profile of the nursing staff } \\
\text { and perceptions of the work } \\
\text { performed in a materials center }\end{array}$ & REME & Qualitativo descritivo & NE6 \\
\hline $\begin{array}{l}\text { Lucon et al., } \\
2017^{20} \\
\text { Brasil }\end{array}$ & $\begin{array}{l}\text { Training of nurses to work } \\
\text { in the central sterile supply } \\
\text { department nurse }\end{array}$ & Revista SOBECC & Qualitativo descritivo & NE6 \\
\hline $\begin{array}{l}\text { Forrester et al., } 2018^{21} \\
\text { Estados Unidos }\end{array}$ & $\begin{array}{l}\text { Surgical instrument } \\
\text { reprocessing in resource- } \\
\text { constrained countries: a } \\
\text { scoping review of existing } \\
\text { methods, policies, and barriers }\end{array}$ & Surgical Infections & Scoping review & NE5 \\
\hline $\begin{array}{l}\text { Wang et al., } \\
2018^{22} \\
\text { China }\end{array}$ & $\begin{array}{l}\text { Application of a sub- } \\
\text { specialties management } \\
\text { model improves quality } \\
\text { control in a central sterile } \\
\text { supply department }\end{array}$ & $\begin{array}{c}\text { BMC Health Services } \\
\text { Research }\end{array}$ & Misto descritivo & NE6 \\
\hline
\end{tabular}


Quadro 3. Continuação.

\begin{tabular}{|c|c|c|c|c|}
\hline Autores, ano e país & Título & Periódico & Delineamento & Nível de evidência \\
\hline $\begin{array}{l}\text { Fast et al., } \\
2018^{23} \\
\text { Benim }\end{array}$ & $\begin{array}{l}\text { Mixed methods evaluation } \\
\text { of the impact of a short term } \\
\text { training program on sterile } \\
\text { processing knowledge, } \\
\text { practice, and attitude in three } \\
\text { hospitals in Benin }\end{array}$ & $\begin{array}{l}\text { Antimicrobial } \\
\text { Resistance and } \\
\text { Infection Control }\end{array}$ & $\begin{array}{l}\text { Retrospectivo } \\
\text { comparativo }\end{array}$ & NE4 \\
\hline $\begin{array}{l}\text { Fast et al., } 2019^{24} \\
\text { Etiópia }\end{array}$ & $\begin{array}{l}\text { The impact of a short-term } \\
\text { training program on workers' } \\
\text { sterile processing knowledge } \\
\text { and practices in } 12 \text { Ethiopian } \\
\text { hospitals: a mixed methods } \\
\text { study }\end{array}$ & PLoS One & Misto descritivo & NE6 \\
\hline
\end{tabular}

Quadro 4. Síntese dos artigos sobre educação em CME publicados em periódicos no período de 2015 a 2020.

\begin{tabular}{|c|c|c|c|}
\hline $\begin{array}{l}\text { Autor(es), ano } \\
\text { e país }\end{array}$ & Objetivo & Aspectos metodológicos & Resultados \\
\hline $\begin{array}{l}\text { Athanázio e } \\
\text { Cordeiro, } \\
2015^{15} \\
\text { Brasil }\end{array}$ & $\begin{array}{l}\text { Analisar como a educação } \\
\text { permanente pode trabalhar } \\
\text { a qualificação do profissional } \\
\text { de enfermagem do CME. }\end{array}$ & $\begin{array}{l}\text { Aplicação de entrevistas } \\
\text { semiestruturadas com análise de } \\
\text { conteúdo pelo método de Bardin. }\end{array}$ & $\begin{array}{l}\text { Artigo Nota Prévia apresenta resultados } \\
\text { esperados de colaborar para melhor } \\
\text { compreensão da Política Nacional de EPS } \\
\text { por parte dos profissionais atuantes no CME; } \\
\text { implantação e desenvolvimento da EPS na } \\
\text { unidade; e futuras reflexões, discussões e } \\
\text { pesquisas acerca da EPS. }\end{array}$ \\
\hline $\begin{array}{l}\text { Tolson e } \\
\text { Friedewald } \\
2016^{16} \\
\text { Austrália }\end{array}$ & $\begin{array}{l}\text { Relatar a construção e } \\
\text { aplicação dos “5 Momentos” } \\
\text { de higiene de mãos no CME. }\end{array}$ & $\begin{array}{l}\text { Descrição da implementação } \\
\text { de um programa de auditoria } \\
\text { associado a atividades } \\
\text { educacionais para o treinamento } \\
\text { de técnica eficaz de higiene de } \\
\text { mãos. }\end{array}$ & $\begin{array}{l}\text { A intervenção promoveu aumento na } \\
\text { conscientização da necessidade de melhorar } \\
\text { as práticas pessoais de higiene de mãos. } \\
\text { A adesão global para prática de higiene de } \\
\text { mãos passou de } 43 \% \text { para } 88 \% \text {. }\end{array}$ \\
\hline $\begin{array}{l}\text { Farias et al., } \\
2016^{17} \\
\text { Brasil }\end{array}$ & $\begin{array}{c}\text { Identificar a percepção dos } \\
\text { profissionais de enfermagem } \\
\text { sobre educação continuada } \\
\text { no CME. }\end{array}$ & $\begin{array}{l}\text { Aplicação de questionário com } \\
\text { perguntas fechadas e objetivas, e } \\
\text { entrevista semiestruturada com } \\
\text { análise do discurso do sujeito } \\
\text { coletivo (DSC). }\end{array}$ & $\begin{array}{l}\text { Observou-se no DSC que o conceito de EC é } \\
\text { compreendido como educação direcionada } \\
\text { para melhoria da atuação do profissional. } \\
\text { Evidenciou-se ausência de atividades de EC e } \\
\text { insegurança da equipe quanto ao domínio de } \\
\text { novas tecnologias. }\end{array}$ \\
\hline $\begin{array}{l}\text { Paula et al., } \\
2017^{18} \\
\text { Brasil }\end{array}$ & $\begin{array}{l}\text { Relatar a experiência da } \\
\text { elaboração de uma cartilha } \\
\text { sobre o processamento de } \\
\text { PPS em Unidades de Atenção } \\
\text { Primária à Saúde (Uaps). }\end{array}$ & $\begin{array}{c}\text { Construção de material } \\
\text { educativo no formato de } \\
\text { cartilha envolvendo três etapas: } \\
\text { preparação do conteúdo baseado } \\
\text { na literatura científica; seleção } \\
\text { de ilustrações em sites de } \\
\text { busca da internet; elaboração e } \\
\text { montagem. }\end{array}$ & $\begin{array}{l}\text { A cartilha foi estruturada em quatro } \\
\text { capítulos: 1. CME: conceitos e requisitos de } \\
\text { infraestrutura; } 2 \text {. Classificação dos artigos } \\
\text { e biossegurança em CME; } 3 \text {. Áreas do CME } \\
\text { e etapas do processamento de PPS; } 4 \text {. } \\
\text { Monitoramento do processo de esterilização. }\end{array}$ \\
\hline $\begin{array}{l}\text { Bugs et al., } \\
2017^{19} \\
\text { Brasil }\end{array}$ & $\begin{array}{l}\text { Traçar o perfil da equipe } \\
\text { de enfermagem, identificar } \\
\text { os processos de EC e as } \\
\text { percepções da equipe do CME } \\
\text { sobre o processo de trabalho } \\
\text { realizado. }\end{array}$ & $\begin{array}{l}\text { Aplicação de questionário } \\
\text { semiestruturado para } \\
\text { levantamento de dados pessoais, } \\
\text { informações de perfil profissional } \\
\text { e valorização do trabalho da } \\
\text { equipe de enfermagem. }\end{array}$ & $\begin{array}{l}\text { Identificaram-se treinamentos relacionados a } \\
\text { atividades técnicas do processo de trabalho, } \\
\text { porém somente } 56,25 \% \text { dos entrevistados } \\
\text { foram treinados ao longo de dois anos de } \\
\text { trabalho no CME. }\end{array}$ \\
\hline
\end{tabular}


Quadro 4. Continuação.

\begin{tabular}{|c|c|c|c|}
\hline $\begin{array}{l}\text { Autor(es), ano } \\
\text { e país }\end{array}$ & Objetivo & Aspectos metodológicos & Resultados \\
\hline $\begin{array}{l}\text { Lucon et al., } \\
2017^{20} \\
\text { Brasil }\end{array}$ & $\begin{array}{c}\text { Compreender a formação } \\
\text { do enfermeiro para atuar no } \\
\text { CME. }\end{array}$ & $\begin{array}{l}\text { Aplicação de entrevistas, com } \\
\text { análise de conteúdo de Bardin. }\end{array}$ & $\begin{array}{c}\text { A análise de conteúdo evidenciou } \\
\text { dificuldades para apreender as práticas em } \\
\text { CME, identificar lacunas de conhecimento } \\
\text { e aprofundar fundamentação teórica. A } \\
\text { construção do aprendizado necessita de } \\
\text { articulação entre teoria e prática com } \\
\text { abordagem abrangente. }\end{array}$ \\
\hline $\begin{array}{l}\text { Forrester et al., } \\
2018^{21} \\
\text { Estados Unidos }\end{array}$ & $\begin{array}{l}\text { Explorar o status atualizado } \\
\text { do reprocessamento de PPS } \\
\text { em países de baixa e média } \\
\text { rendas e categorizar as } \\
\text { barreiras para implementação } \\
\text { de diretrizes e práticas } \\
\text { seguras. }\end{array}$ & $\begin{array}{l}\text { Scoping review com descrição } \\
\text { de métodos atuais, diretrizes e } \\
\text { barreiras para reprocessamento } \\
\text { de PPS em países de baixa e } \\
\text { média rendas. }\end{array}$ & $\begin{array}{c}\text { Observaram-se déficits na estrutura e na } \\
\text { força de trabalho do CME, por causa da } \\
\text { insuficiência de treinamento e educação, } \\
\text { além de desatualização tecnológica e falta de } \\
\text { recursos. }\end{array}$ \\
\hline $\begin{array}{l}\text { Wang et al., } \\
2018^{22} \\
\text { China }\end{array}$ & $\begin{array}{l}\text { Investigar o efeito de um } \\
\text { modelo de gestão de rotina } \\
\text { por subespecialidades no } \\
\text { processamento de PPS no } \\
\text { controle de qualidade e na } \\
\text { satisfação do profissional do } \\
\text { CME. }\end{array}$ & $\begin{array}{l}\text { Utilização de um modelo de } \\
\text { rotina por subespecialidade } \\
\text { associado a treinamento } \\
\text { contínuo da equipe em } \\
\text { comparação ao modelo } \\
\text { tradicional. Aplicação de } \\
\text { questionário para avaliação das } \\
\text { competências profissionais. }\end{array}$ & $\begin{array}{l}\text { A adaptação nas rotinas para processamento } \\
\text { de PPS por subespecialidade demonstrou } \\
\text { melhores resultados em relação ao } \\
\text { aprimoramento de conhecimentos teóricos } \\
\text { e habilidades práticas, refletindo-se na } \\
\text { redução de falhas nos processos e elevando } \\
\text { a satisfação das áreas atendidas. }\end{array}$ \\
\hline $\begin{array}{l}\text { Fast et al., } \\
2018^{23} \\
\text { Benim }\end{array}$ & $\begin{array}{l}\text { Avaliar o impacto } \\
\text { dos treinamentos no } \\
\text { conhecimento, nas } \\
\text { habilidades e nas práticas } \\
\text { profissionais, identificando as } \\
\text { mudanças institucionais no } \\
\text { processamento de PPS. }\end{array}$ & $\begin{array}{l}\text { Aplicação de um curso teórico } \\
\text { seguido de mentoria prática in } \\
\text { loco. Análise da intervenção, por } \\
\text { meio de avaliações hospitalares, } \\
\text { testes de conhecimento e } \\
\text { entrevistas semiestruturadas. }\end{array}$ & $\begin{array}{l}\text { A análise temática das entrevistas identificou } \\
\text { cinco temas-chave relativos à mudança nas } \\
\text { práticas e atitudes dos profissionais após a } \\
\text { intervenção: } 1 \text {. Modificação das coisas que } \\
\text { eram feitas; } 2 \text {. Modificação da maneira de ver } \\
\text { as coisas; } 3 \text {. Agora se presta mais atenção; } \\
\text { 4. Redução de infecções de sítio cirúrgico; } 5 \text {. } \\
\text { Preocupações com recursos. }\end{array}$ \\
\hline $\begin{array}{l}\text { Fast et al., } 2019^{24} \\
\text { Etiópia }\end{array}$ & $\begin{array}{l}\text { Identificar o impacto de um } \\
\text { programa de educação com } \\
\text { foco no reprocessamento } \\
\text { de PPS em } 12 \text { unidades de } \\
\text { saúde da Etiópia. }\end{array}$ & $\begin{array}{l}\text { Aplicação de um programa } \\
\text { de aulas teóricas associado à } \\
\text { capacitação de treinadores e } \\
\text { mentoria in loco. }\end{array}$ & $\begin{array}{l}\text { Os resultados dos testes de conhecimento } \\
\text { demonstraram melhorias significativas na } \\
\text { aquisição de conhecimentos. A melhoria } \\
\text { prática foi estatisticamente significativa } \\
\text { em várias áreas do CME depois da } \\
\text { implementação do programa }\end{array}$ \\
\hline
\end{tabular}

CME: Centro de Material e Esterilização; EPS: Educação Permanente em Saúde; EP: Educação Permanente; EC: Educação Continuada; DSC: Discurso do Sujeito Coletivo; PPS: Produtos para Saúde.

demonstrar resultados efetivos na qualificação das equipes que atuam com PPS em diversos países ${ }^{16,18,22-24}$, são ferramentas para motivação e valorização profissionais ${ }^{19,20,22,23}$. Entretanto, a carência de diretrizes robustas e infraestrutura adequada é obstáculo substancial que impacta a incidência de falhas de processos e afeta diretamente a segurança dos pacientes ${ }^{21,22}$.

Adicionalmente, entre as condições necessárias para uma adequada atuação dos profissionais de CME, a atualização constante e a disponibilização de recursos mínimos para as práticas profissionais foram mencionadas nos dez estudos avaliados como indispensáveis para implementação de melhorias na prática clínica ${ }^{15-24}$. Nesse sentido, o desenvolvimento de competências técnicas para o manejo de PPS pode ser acompanhado e aprimorado por meio de diversificadas intervenções, a se destacar: educação permanente com implementação de materiais didáticos ${ }^{15,18}$, aplicação de programas de treinamento teórico e prático ${ }^{16,19,22-24}$, reestruturação dos processos de trabalho com treinamento técnico ${ }^{16,22,24}$, programas de mentoria educativa in $l o c 0^{24}$ e autorreflexão 
profissional sobre melhores práticas aplicadas aos processos de trabalho ${ }^{15,17,20,21}$.

A educação continuada e a educação permanente, realizadas com uso de cartilhas e outros materiais didáticos, demonstram ser propostas simples e viáveis, que facilitam métodos gerenciais e beneficiam a prática assistencial ${ }^{18}$, e estão alinhadas com a Política Nacional de Educação Permanente em Saúde ${ }^{15}$, podendo ser ponto de partida para estimular ações de aprimoramento dos profissionais do CME. Porém, dados mensurando a relação com melhorias em indicadores assistenciais não foram documentados nos cenários dessas intervenções.

Em relação aos programas de treinamento teórico e prático, os impactos na prática profissional foram acompanhados, constatando-se: implementação de novas atitudes em serviço, mudanças funcionais, implementação de melhores práticas, de acordo com recomendações técnicas, e mudanças na percepção sobre a importância e o resultado do trabalho para qualidade na assistência prestada, impactando a redução de infecções cirúrgicas ${ }^{23,24}$.

A adesão a novas práticas inter-relaciona-se com a compreensão dos benefícios e impactos tangíveis. Mensurar os resultados e compartilhar os dados podem contribuir para melhora da cultura organizacional.

Um estudo conduzido em New South Wales ${ }^{16}$, com intervenção para melhoria da qualidade de higiene de mãos da equipe do CME, atingiu a conformidade dessa prática em relação ao benchmark local, por meio de um programa de auditoria entre pares da própria equipe, com associação de atividades teórico-práticas no formato de educação a distância. O resultado das taxas de higiene de mãos ao longo de 18 meses passou de 43 para $88 \%$ de conformidade, desde o início da implementação do programa. Além desse benefício alcançado, outros adicionais foram relatados pela equipe, como: otimismo, autorresponsabilidade, conscientização e mudança cultural.

Uma intervenção realizada em um hospital da cidade de Suzhou ${ }^{22}$ implementou um modelo de rotina por subespecialidade para processamento de PPS associado a treinamento contínuo da equipe. Os resultados demonstraram que aprimorar o conhecimento teórico e as habilidades práticas, além de contribuir com ganhos na eficiência e na qualidade do serviço prestado, também impacta outras dimensões do exercício profissional, bem como a forma como as demais áreas do serviço de saúde enxergam o CME.

Quatro ${ }^{17,19-21}$ das dez publicações incluídas na análise direcionaram-se para a identificação do perfil dos profissionais inseridos nesse contexto em relação às percepções quanto à educação no CME, lacunas de conhecimento da área e oportunidades de desenvolvimento. Os resultados dos estudos reforçaram a importância de intervenções educativas como ferramenta de capacitação profissional para domínio de novas tecnologias, prestação de serviço de qualidade e impacto favorável na eficácia do processamento de PPS ${ }^{17,19-21}$.

Considerando os resultados do presente estudo, verificaram-se poucas publicações sobre estratégias de educação em CME. Uma limitação pode estar relacionada à padronização dos descritores para busca nas diferentes bases de dados. Considerando as especificidades de cada uma, pode-se supor que usar descritores diferentes para cada base pode ser mais efetivo.

Contudo, ficou evidente, durante o processo de busca, a relevância do tema, frequentemente mencionado como fundamental para promover melhorias na cultura organizacional e nas práticas dos processos desempenhados pelos serviços de saúde, em todos os níveis de atenção, tanto em países desenvolvidos como em países em desenvolvimento ${ }^{16,18,23}$.

\section{CONSIDERAÇÕES FINAIS}

Este artigo buscou analisar as publicações recentes que abordavam a educação em CME. Os artigos selecionados apresentaram baixo nível de evidência e $40 \%$ dos estudos não aplicaram estratégias educativas, mas destinavam-se a identificar as percepções dos profissionais em relação à educação no CME.

Os resultados evidenciaram a importância de fomentar novas pesquisas para identificar as necessidades de capacitação dos profissionais que trabalham no CME e para divulgar as iniciativas de educação existentes, de forma a incentivar o aprimoramento contínuo dessa área vital para os serviços de saúde. Os resultados demonstraram que a implementação de intervenções educativas possibilita o desenvolvimento técnico para domínio de novas tecnologias, reduz falhas no processamento de PPS, melhorando a qualidade do serviço prestado, e estimula o engajamento, a conscientização e a satisfação dos profissionais.

Sugere-se a construção de mais pesquisas que envolvam essa temática, a fim de promover a qualificação e a valorização das equipes que atuam na linha de frente do processamento de PPS. 


\section{REFERÊNCIAS}

1. Associação Brasileira de Enfermeiros de Centro Cirúrgico, Recuperação Anestésica e Centro de Material e Esterilização (SOBECC). Diretrizes de práticas em enfermagem cirúrgica e processamento de produtos para a saúde. 7ª ed. Barueri: Manole; São Paulo: SOBECC; 2017.

2. World Health Organization and Pan American Organization. Decontamination and reprocessing of medical devices for healthcare facilities [Internet]. Genebra: World Health Organization; 2016 [acessado em 4 nov. 2020]. Disponível em: https://apps.who.int/iris/ bitstream/handle/10665/250232/9789241549851-eng.pdf

3. Pereira RA, Morais CM, Santos SJ, Silveira MPM. A atuação do enfermeiro frente às normas e rotinas relacionadas ao processamento de artigos na central de materiais de esterilização. Revista Extensão. 2019 [acessado em 4 nov. 2020];3(1):53-62. Disponível em: https:// revista.unitins.br/index.php/extensao/article/view/1685/1124

4. Ouriques $\mathrm{CM}$, Machado ME. Enfermagem no processo de esterilização de materiais. Texto Contexto Enferm. 2013;22(3):695-703. https:// doi.org/10.1590/S0104-07072013000300016

5. Leite ES, Silva EN, Silva EN, Santos J. Educação continuada na central de material e esterilização: significados e dificuldades enfrentadas pela enfermagem. Rev SOBECC. 2011;16(4):31-9.

6. Espindola MCG, Fontana RT. Riscos ocupacionais e mecanismos de autocuidado do trabalhador de um centro de material e esterilização. Rev Gaúcha Enferm. 2012;33(1):116-23. https://doi.org/10.1590/ S1983-14472012000100016

7. Costa R, Montenegro HRA, Silva RN, Almeida Filho AJ. The role of central sterile supply department nursing team members: an integrative review. Esc Anna Nery. 2020 [acessado em 4 nov. 2020];24(3):e20190316. Disponível em: https://www.scielo.br/j/ ean/a/QnTJBVXYgLKwPQCJgpmzbZp/?lang=en https://doi.org/10.1590/2177-9465-EAN-2019-0316

8. Aguiar BGC, Soares E, Silva AC. Evolução das centrais de material e esterilização: história, atualidades e perspectivas para a enfermagem. Enferm Glob. 2009 [acessado em 4 nov. 2020];(15):1-6. Disponível em: https://scielo.isciii.es/pdf/eg/n15/pt_reflexion2.pdf

9. Tipple AF, Souza TR, BezerraAL, Munari DB. O trabalhador sem formação em enfermagem atuando em centro de material e esterilização: desafio para o enfermeiro. Rev Esc Enferm USP. 2005;39(2):173-80. https:// doi.org/10.1590/S0080-62342005000200007

10. Padoveze MC, Figueiredo RM, Pelaes CES, Otrenti E. Necessidades de aprendizagem de enfermeiros sobre processos de esterilização. Rev SOBECC. 2013;18(3):23-9.

11. Mendes KDS, Silveira RCCP, Galvão CM. Revisão integrativa: método de pesquisa para a incorporação de evidências na saúde e na enfermagem. Texto Contexto Enferm. 2008;17(4):758-64. https:// doi.org/10.1590/S0104-07072008000400018

12. Grupo Anima Educação Manual de revisão bibliográfica sistemática integrativa: a pesquisa baseada em evidências. Belo Horizonte: Grupo Anima Educação; 2014 [acessado em 26 jul. 2020]. Disponível em: http://biblioteca.cofen.gov.br/wp-content/uploads/2019/06/manual_ revisao_bibliografica-sistematica-integrativa.pdf
13. Moher D, Liberati A, Tetzlaff J, Altman DG, the PRISMA group. Preferred reporting items for systematic reviews and meta-analyses: the PRISMA statement. PLoS Med. 2009 [acessado em 18 set. 2020];6(7):e1000097. Disponível em: https://journals.plos.org/ plosmedicine/article?id=10.1371/journal.pmed. 1000097 https://doi.org/10.1371/journal.pmed.1000097

14. Melnyk BM, Fineout-Overholt E. Evidence based practice in nursing \& healthcare: a guide to best practice. $3^{a}$ ed. Filadélfia: Wolters Kluwer Health/ Lippincott Williams \& Wilkins; 2015.

15. Athanázio AR, Cordeiro BC. Educação permanente a trabalhadores da central de material e esterilização. Rev Enferm UFPE on line. 2015;9(6):8758-61. https://doi. org/10.5205/1981-8963-v9i6a10657p8758-8761-2015

16. Tolson K, Friedewald M. Beyond the patient zone: improving hand hygiene performance in a sterilising services department. Infect Dis Health. 2016;21:11-5. http://dx.doi.org/10.1016/j.idh.2016.01.003

17. Farias IP, Caldas CM, Miranda LN, Nagliate PC, Freitas DA, Vasconcelos EL. Continuing education in a material and sterilization center: perception of the nursing team. Rev Enferm UFPE on line. 2016;10(7):2604-10. http://dx.doi.org/10.5205/1981-8963-v10i7al1320p2604-2610-2016

18. Paula FMS, Beserra NCN, Lopes RCS, Guerra DR. Elaboration of didactic material for processing health products in primary health care units. Rev SOBECC. 2017;22(3):165-70. http://dx.doi.org/10.5327/ Z1414-4425201700030008

19. Bugs TV, Rigo DFH, Bohrer CD, Borges F, Marques LGS, Vasconcelos $\mathrm{RO}$, et al. Profile of the nursing staff and perceptions of the work performed in a materials center. REME Rev Min Enferm. 2017;21:e996. http://dx.doi.org/10.5935/1415-2762.20170006

20. Lucon SMR, Braccialli LAD, Pirolo SM, Munhoz CC. Training of nurses to work in the central sterile supply department nurse. Rev SOBECC. 2017;22(2):90-7. https://doi.org/10.5327/ Z1414-4425201700020006

21. Forrester JA, Powell BL, Forrester JD, Fast C, Weiser TG. Surgical instrument reprocessing in resource-constrained countries: a scoping review of existing methods, policies, and barriers. Surg Infect. 2018;19(6):593-602. https://doi.org/10.1089/sur.2018.078

22. Wang L, Cai X, Cheng P. Application of a sub-specialties management model improves quality control in a central sterile supply department. BMC Health Serv Res (Online). 2018;18(1):385. https://doi.org/10.1186/ s12913-018-3214-7

23. Fast O, Fast C, Fast D, Veltiens S, Salami Z, White M. Mixed methods evaluation of the impact of a short term training program on sterile processing knowledge, practice, and attitude in three hospitals in Benin. Antimicrob Resist Infect Control. 2018;7:20. https://doi. org/10.1186/s13756-018-0312-6

24. Fast MO, Teka HG, Alemayehu/Gebreselassie M, Fast CMD, Fast D, Uzoka FME. The impact of a short-term training program on workers' sterile processing knowledge and practices in 12 Ethiopian hospitals: a mixed methods study. PLoS One. 2019;14(5):e0215643. https:// dx.doi.org/10.1371\%2Fjournal.pone.0215643 